\title{
Capilaroscopia Periungueal Seriada (CPU) como Parâmetro de Monitoramento da Evolução de Pacientes com Diagnóstico Inicial de Fenômeno de Raynaud (FRy) Isolado ou de Doença Indiferenciada do Tecido Conjuntivo (DITC)
}

\section{Serial Nailfold Capillaroscopy (NFC) as a Tool for Prospective Evaluation of Patients with initial Isolated Raynaud's Phenomenon (RP) or Undifferentiated Connective Tissue Disease (UCDT)}

\author{
Viviane Shinsato Higashi( ${ }^{(1)}$, Cristiane Kayser ${ }^{(2)}$, Luís Eduardo C. Andrade ${ }^{(3)}$
}

\section{RESUMO}

Objetivos: Análise evolutiva retrospectiva de alterações capilaroscópicas e correlação com a evolução diagnóstica em pacientes com quadro inicial de fenômeno de Raynaud (FRy) isolado ou de doença indiferenciada do tecido conjuntivo (DITC). Métodos: Foram avaliados 19 pacientes com diagnóstico pregresso de FRy isolado ou de DITC com duas ou mais capilaroscopias periungueais (CPUs) realizadas com intervalo de pelo menos um ano entre elas. Resultados: Dos 19 pacientes, 11 tinham diagnóstico inicial de FRy isolado sendo que na segunda avaliação, sete mantiveram o mesmo diagnóstico, três evoluíram para esclerose sistêmica (ES) e um para síndrome de Sjögren $(\mathrm{SSj})$. Oito pacientes tinham diagnóstico inicial de DITC, sendo que todos permaneceram com o mesmo diagnóstico. Nove pacientes apresentaram mudanças no padrão da CPU. Em sete, houve piora do padrão capilaroscópico, com aparecimento ou acentuação do padrão SD (Grupo A); três deles tiveram evolução do diagnóstico de FRy para ES. Os quatro casos restantes (três com DITC e um com FRy isolado) mantiveram seus diagnósticos. Em dois pacientes houve melhora da CPU (Grupo B); um deles evoluiu de diagnóstico de FRy isolado para SSj. Em dez pacientes a CPU permaneceu estável (Grupo C); todos mantiveram o diagnóstico inicial (quatro com DITC, seis com FRy). Todos os três pacientes que evoluíram para ES apresentaram aparecimento ou acentuação da microangiopatia SD (sensibilidade de 100\%). Entre os 16 casos que não evoluíram para ES, 12 não apresentaram aparecimento ou acentuação da microangiopatia SD (especificidade de 75\%). Conclusões: $\mathrm{O}$ aparecimento ou acentuação do padrão SD no intervalo de tempo estudado teve alta sensibilidade e moderada especificidade na evolução para o diagnóstico de ES.

Palavras-chave: capilaroscopia periungueal, microcirculação, esclerose sistêmica, fenômeno de Raynaud.

\begin{abstract}
Objective: Retrospective serial analysis of nailfold capillaroscopy (NFC) alterations and its correlation with diagnostic evolution in patients initially presenting isolated Raynaud's phenomenon $(R P)$ or undifferentiated connective tissue disease (UCTD). Methods: We evaluated 19 patients with previous diagnosis of isolated Raynaud's Phenomenon or UCTD who had two or more NFC exams within at least one-year interval. Results: Eleven patients have had initial diagnosis of isolated RP; seven maintained the RP diagnosis, three developed Systemic Sclerosis (SSc), and one evolved to Sjögren's Syndrome (SSj). Eight patients were diagnosed with UCTD in the first evaluation and all of them kept the initial diagnosis. Nine patients presented changes in NFC pattern. Seven patients presented intensification of SD pattern (group A). In three of these patients, the diagnosis has evolved from isolated FRy to SSc. The remaining four cases (one $R P$ and three UCTD) maintained the initial diagnosis. Group B included two cases in which the SD pattern has been attenuated or disappeared. One of these patients has evolved from isolated RP to SSj. Ten patients had the NFC pattern unaltered (Group C) and all of them have kept the initial diagnosis (four with UCTD and six with RP). All patients who had evolved to SSc have shown intensification or appearance of SD pattern (sensitivity=100\%). On the other hand, among the 16 patients who have not evolved to SSc, 12 had not shown appearance or intensification of SD pattern (specificity=75\%). Conclusions: The appearance or intensification of SD pattern in serial NFC analysis during the study period has shown high sensitivity and moderate specificity in regard to the development of SSc.
\end{abstract}

Keywords: nailfold capillaroscopy, microcirculation, systemic sclerosis, Raynaud's phenomenon.

Disciplina de Reumatologia, Escola Paulista de Medicina, Universidade Federal de São Paulo (UNIFESP). Recebido em 17/10/2005. Aprovado, após revisão, em 20/1 1/2005.

1. Aluna de graduação do curso médico, UNIFESP. $\quad 2$. Médica Doutora Associada da Disciplina de Reumatologia da UNIFESP.

3. Professor Adjunto - Livre Docente, Disciplina de Reumatologia da UNIFESP.

Endereço para correspondência: Luís E.C. Andrade, Rua Botucatu, 740, $3^{\circ}$ andar, São Paulo, CEP 04023 062, SP, Brasil, e-mail: luis@reumato.epm.br 


\section{INTRODUÇÃO}

O fenômeno de Raynaud (FRy) caracteriza-se por episódios reversíveis de vasoespasmos associados a alterações de coloração típica das mãos e/ou pés, usualmente após contato com o frio ou estresse emocional. A alteração de coloração ocorre classicamente em três fases sucessivas: palidez, cianose e rubor. O FRy é relativamente freqüente, acometendo de 4 a $15 \%$ da população geral ${ }^{(1,3)}$. Em cerca de 90\% dos casos, caracteriza-se por ser um evento funcional benigno, não havendo qualquer doença subjacente, sendo chamado assim de FRy primário ou idiopático. O FRy é chamado de secundário quando está associado a alguma doença ou condição subjacente, sendo a esclerose sistêmica (ES) a enfermidade em que o FRy é mais freqüente. Além da ES, pode ocorrer em diversas outras doenças reumáticas auto-imunes, doenças mieloproliferativas, crioglobulinemia, arteriopatias obstrutivas, ou ser induzido por fármacos ${ }^{(4,5)}$.

As doenças do espectro da ES têm muitas vezes início insidioso e paucissintomático. O FRy costuma ser o sintoma inicial mais freqüente nesses casos, podendo preceder por vários meses ou anos os outros sinais e sintomas da doença $^{(6)}$. Sendo assim, muitos desses pacientes são acompanhados nos ambulatórios de Reumatologia durante vários anos, inicialmente com diagnóstico de FRy idiopático ou doença indiferenciada do tecido conjuntivo (DITC), pois não preenchem os critérios diagnósticos para uma doença reumática específica no início do quadro. Nesses casos, seria interessante podermos lançar mão de alguma ferramenta que auxilie na seleção dos pacientes que desenvolverão uma doença reumática auto-imune, especialmente a ES, permitindo um diagnóstico precoce ou que seja útil na definição do prognóstico desses pacientes.

A capilaroscopia periungueal (CPU) é um exame nãoinvasivo, de fácil execução, considerado um método confiável para a identificação de alterações morfológicas da microcirculação, que estão associadas com as doenças do espectro da $\mathrm{ES}^{(7)}$. No FRy primário ou idiopático, a CPU apresenta um padrão semelhante ao normal, ou seja, presença de alças capilares de tamanho, forma e cor homogêneas, dispostas transversalmente ao longo da cutícula. Eventualmente, alguns desses pacientes apresentam alguns capilares discretamente ectasiados, mas tipicamente há ausência de outras alterações morfológicas. Já os pacientes com FRy associado a doenças do espectro da ES apresentam um quadro microangiopático específico, denominado padrão SD, caracterizado pela presença de dilatação (ectasia) e distorção capilar, associado a áreas de completa desvas- cularização, além de diminuição difusa da quantidade de alças capilares ${ }^{(8,9)}$.

Alguns pacientes com diagnóstico inicial de FRy isolado ou de DITC podem apresentar o padrão SD à CPU e acredita-se que isto seja um fator prognóstico sugestivo de evolução para ES. Não existem, entretanto, estudos na literatura acompanhando evolutivamente as alterações capilaroscópicas e clínicas nesses dois grupos de pacientes. Não se sabe, portanto, o significado clínico e prognóstico de alterações progressivas na CPU nesse grupo de pacientes.

Nesse contexto, realizamos uma análise evolutiva retrospectiva de alterações capilaroscópicas em pacientes com quadro inicial de FRy isolado ou de DITC e confrontamos a evolução das alterações à CPU com a evolução diagnóstica desse grupo de pacientes ao longo do acompanhamento.

\section{MATERIAIS E MÉTODOS}

Foram avaliados 19 pacientes oriundos do ambulatório de Reumatologia do HSP-UNIFESP com diagnóstico pregresso de FRy isolado ou de DITC que apresentavam também FRy. Para o diagnóstico de FRy isolado, utilizamos os critérios propostos por LeRoy et al ${ }^{(10)}$ para o diagnóstico de FRy idiopático, mas com exclusão da CPU dos mesmos, por motivos óbvios. Estes incluem a presença de pulsos periféricos fortes e simétricos, ausência de úlcera ou gangrena digital, capilaroscopia periungueal normal, ausência de anticorpos antinúcleo (FAN) e velocidade de hemossedimentação normal. Os pacientes com diagnóstico de DITC apresentavam, além do FRy, outros sinais e/ou sintomas sugestivos de doença reumática auto-imune (artrite, artralgia, presença de FAN, etc.), mas não preenchiam critérios de classificação para ES ou qualquer outra doença reumática auto-imune. Os critérios de classificação foram aqueles preconizados pelo American College of Rheumatology (ACR).

Todos os pacientes deveriam ter realizado dois ou mais exames capilaroscópicos com intervalo de pelo menos um ano entre eles. Em pacientes que tinham apenas uma CPU realizada há mais de um ano, uma nova CPU foi realizada para avaliação seqüencial da microangiopatia periungueal. Todos os pacientes que foram submetidos a uma nova CPU deveriam ter maioridade e ter assinado o termo ético de consentimento, aprovado pela Comissão de Ética da UNIFESP.

Avaliamos a evolução do diagnóstico de cada paciente e confrontamos com a evolução da CPU. Os parâmetros relevantes para o diagnóstico de ES (segundo os critérios 
do $A C R)^{(11)}$, bem como os parâmetros capilaroscópicos, foram coletados diretamente do prontuário do paciente. Os dados clínicos de cada paciente foram coletados da primeira consulta realizada e da consulta mais próxima da última CPU. Os dados sorológicos coletados resultam de exames feitos no período entre as consultas analisadas.

Resumidamente, a capilaroscopia periungueal foi realizada seguindo-se um protocolo padrão, no qual todos os dez dígitos de ambas as mãos foram analisados utilizando-se um Estereomicroscópio SZ40 (Olympus ${ }^{\circledR}$ ) com câmera de captação de imagens acoplada. Foram utilizados os seguintes parâmetros para avaliação da CPU:

1) número de alças por $\mathrm{mm}$;

2) número de capilares ectasiados, que correspondem a alças alargadas em todos os seus três ramos - aferente, transição e eferente, correspondendo a cerca de quatro vezes maior que o normal; e número de megacapilares, que correspondem a alças extremamente alargadas com calibre dez vezes maior que as alças adjacentes normais ${ }^{(12)}$;

3) escore de deleção capilar: para quantificar o grau de deleção, ou seja, a magnitude de áreas avasculares. Seguimos a escala de Lee et $a l^{(9)}$, que definem como deleção a ausência de dois ou mais capilares sucessivos, permitindo quantificar este parâmetro numa escala de 0 a 3 conforme a extensão das lesões: 0 - nenhuma área de deleção; 1 - uma ou duas áreas de deleção descontínuas; 2 - mais de duas áreas de deleção descontínuas; 3 - áreas extensas e confluentes de desvascularização.

Para análise dos diversos parâmetros, o número de capilares $/ \mathrm{mm}$, de capilares ectasiados e de megacapilares e o grau de deleção foram compilados como a média obtida nos dedos analisados. Para considerarmos que houve melhora ou piora capilaroscópica, uma das seguintes alterações entre os dois exames seriados deveriam estar presentes: aumento ou diminuição de no mínimo dois capilares $/ \mathrm{mm}$, alteração no número médio de capilares ectasiados maior que 0,5 , alteração no número médio de megacapilares maior que 0,1 , ou alteração maior que 0,1 no escore de deleção. O estabelecimento dos critérios para melhora ou piora capilaroscópica, acima detalhados, baseou-se na experiência acumulada de cerca de 20 anos dos pesquisadores, já que não existem estudos na literatura avaliando evolutivamente os parâmetros empregados no presente estudo. A análise dos registros com os dados obtidos pelas CPUs foi feita de forma independente por dois observadores afeitos à interpretação do exame, sem conhecimento prévio da evolução clínica do paciente. Quando houve discordância entre as duas avaliações, os dois observadores fizeram nova avaliação em conjunto para chegarem a um consenso.

\section{ANÁLISE ESTATÍSTICA}

Para análise dos tempos entre a primeira e a segunda avaliação capilaroscópica nos pacientes que apresentaram e não apresentaram mudanças no padrão da CPU, foi utilizado o teste de Mann-Whitney. Para análise da proporção de pacientes que evoluíram para ES em função da mudança no padrão da CPU, utilizamos o teste de Fisher. Adotamos o nível de significância de $5 \%$ para a conclusão de todos os testes realizados.

\section{RESULTADOS}

Foram avaliados 19 pacientes (18 do sexo feminino e um do masculino) entre o período de 1988 a 2003. A idade média dos pacientes na primeira consulta foi de 35,7 anos, variando de 14 a 67 anos.

A média de tempo do aparecimento do FRy nos 19 pacientes foi de 2,7 anos, variando de um a dez anos. O tempo médio entre a primeira e a segunda avaliação foi de 4,9 anos, variando de 2 a 14 anos. Nos pacientes que apresentaram mudanças no padrão da CPU, o tempo médio entre a primeira e a segunda avaliação foi de 5,8 anos. Nos que mantiveram o padrão capilaroscópico inicial, o tempo médio entre a primeira e a segunda avaliação foi de 4,2 anos. Não houve diferença estatisticamente significante no tempo médio entre as avaliações entre esses dois grupos $(p=0,43)$.

\section{EVOLUÇÃO DO DIAGNÓSTICO DOS PACIENTES}

Entre os 19 pacientes avaliados, 11 tinham diagnóstico inicial de FRy isolado e oito de DITC. Os pacientes com FRy tinham pesquisa do FAN negativa. Nos oito pacientes com DITC, todos apresentavam positividade na pesquisa do FAN. Anti-SSA/Ro foi detectado em quatro pacientes e o anti-RNP em cinco pacientes. Anti-SSB/La e anti-Sm foram negativos em todos os casos. A pesquisa de anticorpo anticentrômero foi negativa nos oito pacientes. Ao exame clínico inicial, dois pacientes apresentavam esclerodactilia e nenhum apresentou microcicatrizes em polpas digitais, ulcerações digitais ou telangiectasias.

Na segunda avaliação, entre os 11 pacientes com diagnóstico inicial de FRy isolado, sete permaneceram com o mesmo diagnóstico, três evoluíram para diagnóstico de ES e um teve diagnóstico de síndrome de Sjögren. Entre os oito pacientes com diagnóstico inicial de DITC, todos permaneceram com o mesmo diagnóstico. 


\section{EVOLUÇÃO DA CPU}
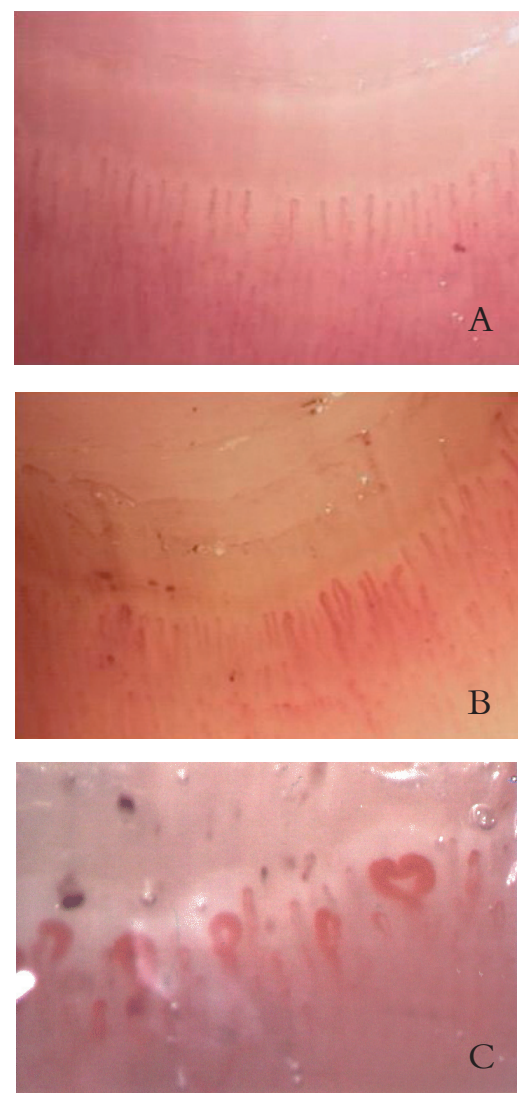

Figura 1 - Fotografias ilustrativas mostrando aspecto de uma Capilaroscopia periungueal normal: $(A)$ alça de forma e distribuição homogêneas, com alterações que correspondem à microangiopatia não específica; (B) presença de alguns capilares ectasiados; ausência de desvascularização e alterações características do padrão SD; (C) capilares gigantes e áreas de desvascularização

Entre os 19 pacientes analisados, oito (42\%) apresentavam CPU normal (Figura 1A) no primeiro exame realizado. Onze apresentavam algum grau de microangiopatia, sendo que quatro apresentavam microangiopatia não específica (Figura 1B) e sete apresentavam padrão SD (Figura 1C). Nos pacientes com CPU normal na avaliação inicial, quatro tinham diagnóstico de FRy isolado e quatro de DITC.

Nove pacientes (47\%) apresentaram mudanças no padrão da CPU e dez mantiveram o padrão inicial. Entre os nove pacientes que apresentaram mudança na CPU, sete pacientes evidenciaram piora do padrão capilaroscópico, com aparecimento ou acentuação do padrão SD e dois registraram melhora da CPU. Esses dois últimos apresentavam um quadro de microangiopatia incipiente e evoluíram para um padrão capilaroscópico normal ao final do acompanhamento (Tabela 1).
TABELA 1

EVOLUÇÃO NOS PARÂMETROS DA CAPILAROSCOPIA PERIUNGUEAL (CPU) EM PACIENTES COM DIAGNÓSTICO INICIAL DE FENÔMENO DE RAYNAUD (FRY) ISOLADO OU DOENÇA INDIFERENCIADA DO TECIDO CONJUNTIVO (DITC)

\begin{tabular}{lccc}
\hline & \multicolumn{3}{c}{ Evolução da CPU } \\
\hline & Estável & Piora & Melhora \\
\hline $\begin{array}{l}\text { Diagnóstico inicial de } \\
\text { FRy isolado }(\mathrm{n}=11)\end{array}$ & 6 & 4 & 1 \\
$\begin{array}{l}\text { Diagnóstico inicial } \\
\text { de DITC }(\mathrm{n}=8)\end{array}$ & 4 & 3 & 1 \\
\begin{tabular}{l} 
Total $(\mathrm{n}=19)$ \\
\hline
\end{tabular} & 10 & 7 & 2 \\
\hline
\end{tabular}

CONFRONTO ENTRE A EVOLUÇÃo DIAGNÓSTICA E A EVOLUÇÃO DA CPU

No grupo de pacientes que apresentou piora da CPU (Grupo A), observou-se evolução do diagnóstico de FRy para ES em três casos. Dois desses pacientes que evoluíram para ES apresentavam uma microangiopatia incipiente na CPU inicial e evoluíram para padrão SD bem definido ao final do acompanhamento; o terceiro apresentava CPU inicial normal e evoluiu para um padrão de microangiopatia não específica após três anos de acompanhamento. Os quatro casos restantes (três com diagnóstico de DITC e um com diagnóstico de FRy isolado) tiveram seus diagnósticos iniciais conservados (Tabela 2).

TABELA 2

DISTRIBUIÇÃO DO NÚMERO DE PACIENTES CONFORME EVOLUÇÃO DIAGNÓSTICA E A EVOLUÇÃO DA CAPILAROSCOPIA PERIUNGUEAL

\begin{tabular}{lcccccccccccc}
\hline & CPU estável & $\begin{array}{c}\text { Piora } \\
\text { capilaroscópica }\end{array}$ & \multicolumn{4}{c}{$\begin{array}{c}\text { Melhora } \\
\text { capilaroscópica }\end{array}$} \\
\hline Diagnóstico* & FRy & DITC & ES & FRy & DITC & ES & FRy & DITC & ES & SSj \\
Inicial & 6 & 4 & 0 & 4 & 3 & 0 & 1 & 1 & 0 & 0 \\
Final & 6 & 4 & 0 & 1 & 3 & 3 & 0 & 1 & 0 & 1 \\
\hline
\end{tabular}

*FRy: Fenômeno de Raynaud; DITC: Doença Indiferenciada do Tecido Conjuntivo; ES: Esclerose Sistêmica; SSj: Síndrome de Sjögren

Nos dois casos em que houve melhora da CPU (Grupo B), um evoluiu de diagnóstico inicial de FRy isolado para SSj e o outro permaneceu estável. Não se observou nesse grupo a evolução para diagnóstico de ES. Nos dez casos em que a CPU permaneceu estável (Grupo C), todos também mantiveram o diagnóstico inicial (quatro com diagnóstico de DITC e seis com diagnóstico de FRy isolado) (Tabela 2). 
Todos os três pacientes que evoluíram para ES apresentaram aparecimento ou acentuação da microangiopatia SD (sensibilidade de 100\%). Por outro lado, entre os 16 casos que não evoluíram para ES, 12 não apresentaram aparecimento ou acentuação da microangiopatia SD (especificidade de 75\%). A análise desta distribuição pelo teste de Fisher mostrou $\mathrm{p}=0,055$.

\section{DISCUSSÃO}

A ES é uma doença sistêmica auto-imune de etiopatogenia desconhecida, que pode ser considerada como um espectro contínuo, em que vários fenótipos clínicos são observados, desde anormalidades biológicas assintomáticas até um fenótipo de doença grave com risco à vida do paciente ${ }^{(13)}$. A ES costuma também ter um curso progressivo e muitas vezes indolente, especialmente suas fases iniciais.

Muitos pacientes se apresentam inicialmente com quadro de FRy isolado ou DITC e, somente após anos de acompanhamento, estabelece-se o diagnóstico de ES. Acredita-se que até $10 \%$ dos casos inicialmente considerados como FRy primário irão desenvolver uma doença reumática autoimune. Características morfológicas precoces da ES que poderiam auxiliar na detecção desses casos incluem lesões microvasculares e inflamação perivascular, que precedem a fibrose de pele e dos órgãos internos às vezes por vários anos $^{(14)}$. Nesse contexto, a CPU permite análise de componentes morfológicos da microcirculação e tem se mostrado útil na investigação de pacientes com FRy isolado e DITC pela sua capacidade de reconhecimento precoce de casos de ES e síndromes correlatas.

Além da CPU, a pesquisa de auto-anticorpos (FAN), usando células HEp-2 como substrato, também é um exame útil para a investigação desses pacientes. Uma série de estudos prospectivos com indivíduos com FRy demonstra que achados anormais à CPU e a presença de determinados auto-anticorpos em altos títulos identificam um grupo de pacientes com alto potencial de desenvolver sinais e sintomas de $\mathrm{ES}^{(15-17)}$. A CPU e a pesquisa de auto-anticorpos com células HEp-2 como substrato permitem a detecção de cerca de $3 / 4$ de todos os pacientes que passam por uma transição para $\mathrm{ES}^{(18)}$.

A detecção precoce de uma doença tem muitas vantagens, incluindo a oportunidade de se impedir ou de diminuir a sua progressão e de maior compreensão da etiopatogenia da mesma. Embora não haja terapia curativa ou preventiva para a ES, o seu reconhecimento precoce é importante para monitorar e propiciar intervenção terapêutica preco- ce no tratamento de suas manifestações. Acometimento pulmonar é atualmente a causa mais comum de óbito em pacientes com ES. Para a alveolite fibrosante, a intervenção terapêutica com ciclofosfamida em pacientes com a doença em fases iniciais pode ser benéfica ${ }^{(19)}$. Para o tratamento da hipertensão pulmonar, novas drogas como os análogos das prostaglandinas e os inibidores do receptor da endotelina-1 têm se mostrado eficazes. Essas duas classes de agentes têm se mostrado eficazes também para o tratamento do FRy e de úlceras isquêmicas nesses doentes ${ }^{(20,21)}$. Nos casos de crise renal esclerodérmica, o uso precoce de inibidores da enzima conversora de angiotensina (IECA) proporcionou redução importante na mortalidade por essa causa.

Assim como a procura de critérios e exames subsidiários com sensibilidade e especificidade altas para o diagnóstico precoce de ES, a observação seriada do que ocorre no complexo processo de evolução patológica das doenças do espectro da ES também é de extrema importância.

No presente estudo foi observado que $47 \%$ dos pacientes avaliados apresentaram mudanças no padrão capilaroscópico. Isso reflete e corrobora a hipótese de que estas doenças podem ter uma evolução dinâmica e leva ao questionamento sobre a conduta de se realizar reavaliações, regularmente ou não, para rastreamento de doenças do tecido conjuntivo em pacientes com FRy sem quaisquer outras manifestações. Em pacientes com FRy, na presença de anormalidades capilaroscópicas, essas avaliações regulares para a detecção das formas não idiopáticas já é recomendada. Os resultados aqui relatados reforçam esta recomendação e sugerem que a reavaliação capilaroscópica possa ter lugar nesse monitoramento.

Devemos ressaltar que o presente estudo apresenta algumas limitações, como o pequeno número de pacientes avaliados e o fato de ser retrospectivo. Mesmo assim, a análise evolutiva retrospectiva de alterações capilaroscópicas e confronto com a evolução diagnóstica em pacientes com quadro inicial de FRy isolado ou DITC nos permitiu observar a associação entre a evolução capilaroscópica da microangiopatia SD no paciente e a evolução do diagnóstico para ES. Os resultados mostraram que a evolução do diagnóstico para ES é acompanhada de uma acentuação ou aparecimento de quadro microangiopático associado à ES, ou seja, é acompanhada de alterações na microcirculação da região periungueal que compreendem dilatação capilar, diminuição do número de alças e áreas de desvascularização (sensibilidade de 100\%). Ressaltamos também que apenas três pacientes com diagnóstico inicial de FRy isolado ou DITC evoluíram para ES, sendo que a maioria dos pacientes 
permaneceram com o mesmo diagnóstico no momento da última avaliação, o que corrobora observações prévias da literatura. Três quartos dos pacientes que não apresentaram evolução para ES não apresentaram acentuação ou aparecimento do padrão SD (especificidade de 75\%). Além disso, todos os pacientes que não apresentaram mudança na CPU ao final do estudo mantiveram também o diagnóstico inicial.

Os resultados apresentados evidenciam relações evolutivas que podem auxiliar no acompanhamento do paciente, demonstrando que a microcirculação desses pacientes sofre

\section{REFERÊNCIAS}

1. Maricq HR, Weinrich MC, Keil JE, LeRoy EC: Prevalence of Raynaud's phenomenon in the general population. A preliminary study by questionnaire. J Chronic Dis 39: 423-7, 1986.

2. O'Keefe ST, Tsapatsaris NP, Beetham Jr WP: Colour chart assisted diagnosis of Raynaud's phenomenon in an unselected hospital employee population. J Rheumatol 19: 1415-7, 1992.

3. Weinrich MC, Maricq HR, Keil JE, McGregor AR, Diat F: Prevalence of Raynaud's phenomenon in the adult population of South Carolina. J Clin Epidemiol 43: 1343-9, 1990.

4. Maricq HR, McGregor AR, Diat F, et al: Major clinical diagnoses found among patients with Raynaud's phenomenon from the general population. J Rheumatol 17: 1171-6, 1990.

5. Belch J: Raynaud's phenomenon. Cardiovascular Research 33: 25-30, 1997.

6. Seibold JR, Smith EA, Leroy C, Steen VD: Systemic sclerosis. In: Klippel JH, Dieppe PA: Rheumatology, London, Mosby-year book Europe limited, 1994.

7. Andrade LEC, Gabriel A, Assad RL, Ferrari AJL, Atra E: Panoramic Nailfold Capillaroscopy: a New Reading Method and Normal Range. Semin Arthritis Rheum 20: 21-31, 1990.

8. Maricq HR, LeRoy EC, D'Angelo WA, et al: Diagnostic potential of in vivo capillary microscopy in scleroderma and related disorders. Arthritis Rheum 23: 183-189, 1980.

9. Lee P, Leung F, Alderdice C, Armstrong SK: Nailfold capillary microscopy in the connective tissue diseases: a semiquantitative assessment. J Rheumatol 10: 930, 1983.

10. LeRoy EC, Medsger Jr TA: Raynaud's phenomenon: a proposal for classification. Clin Exp Rheumatol 10: 485-8, 1992.

11. Masi AT, Rodnan GP, Medsger Jr TA, et al: Preliminary criteria for alterações dinâmicas ao longo do tempo. Os resultados mostram também que tais alterações podem guardar correlação com o próprio processo patológico da doença ou com o desenvolvimento de outras manifestações em órgãos internos que permitam, então, o diagnóstico de ES. Os dados atuais sugerem um importante papel para a CPU seriada no monitoramento de pacientes com diagnóstico inicial de FRy isolado ou DITC. Entretanto, fazem-se necessários estudos prospectivos com um maior número de doentes para a confirmação dos achados e generalização das conclusões. the classification of systemic sclerosis (scleroderma). Arthritis Rheum 23: 581-90, 1980

12. Maricq HR: Widefield capillary microscopy. Technique and rating scale for abnormalities seen in scleroderma and related disorders. Arthritis Rheum 24: 1159-65, 1981.

13. Wigley FM: When is Scleroderma really Scleroderma? J Rheumatol 28: 1471-3, 2001.

14. Carpentier PH, Maricq HR: Microvasculature in systemic sclerosis. Rheum Dis Clin North Am 16: 75-91, 1990.

15. Weiner ES, Hildebrandt S, Senécal J, et al: Prognostic significance of anticentromere antibodies and anti-topoisomerase I antibodies in Raynaud's disease. Arthritis Rheum 34: 68-77, 1991.

16. Priollet P, Vayssairat M, Housset E: How to classify Raynaud's phenomenon. Am J Med 83: 494-8, 1987.

17. Luggen M, Belhorn L, Evans T, Fitzgerald O, Spencer-Green G: The evolution of Raynaud's phenomenon: a longterm prospective study. J Rheumatol 22: 2226-32, 1995.

18. LeRoy EC, Medsger Jr TA: Criteria for the Classification of Early Systemic Sclerosis. J Rheumatol 28: 1573-76, 2001.

19. Akesson A: Cyclophosphamide therapy for scleroderma. Current Op Rheumatol 10: 579-83, 1998.

20. Scorza R, Caronni M, Mascagni B, et al: Effects of long-term cyclic iloprost therapy in systemic sclerosis with Raynaud's phenomenon. A randomized, controlled study. Clin Exp Rheumatol 19: 503-8, 2001.

21. Ramos-Casals M, Brito-Zeron P, Nardi N, et al: Successful treatment of severe Raynaud's phenomenon with bosentan in four patients with systemic sclerosis. Rheumatology (Oxford) 43: 1454-6, 2004.

22. Mannarino E, Pasqualini L, Fedeli F, Scricciolo V, Innocente S: Nailfold Capillaroscopy in the Screening and Diagnosis of Raynaud's Phenomenon. Angiology 45: 37-42, 1994. 